\title{
Modelling curvelet based signal processing problems via wavelet analysis
}

\author{
Bharat Bhosale \\ Professor SambhajiraoKadam College, Deur-Satara, (M.S.), India \\ Email:bnbhosale15@gmail.com
}

\begin{abstract}
Until recently, the wavelet transform has been used for Mathematical analysis and signal processing problems. But it suffers from the disadvantage of poor directionality, which has undermined its usage in many applications. The curvelet transform is a new extension of the wavelet transform, which aims to deal with intersecting phenomena occurring along curved edges in 2-D signals/images. In this work, a strong relationship between the curvelet and wavelet transforms has been established. This version of a wavelet based curvelet has been exploited to develop a full-fledged analytical framework, presenting it as an extension of well established wavelets. Due to computational complexity, instead of applying a curvelet directly, the curvelet in terms of wavelets has been employed more conveniently in the proposed signal denoising model. Finally, the performance factor analysis is performed on multispectral sample radar image data to demonstrate the efficiency of the proposed model. Besides computational gain, the proposed model shows better performance than the other signal processing models. Proposed model is equally applicable to both pulse signals and digital images.
\end{abstract}

Keywords: Wavelet transform, curvelet transform, signal processing, thresholding 


\section{INTRODUCTION}

Multiresolution techniques are deeply related to signal/image processing, biological and computer vision detection, scientific computing, optical data analysis (Bhosale and Biswas, 2013). In these techniques, wavelet functions are used as a basis with an objective to specify the signal as a collection of its successive approximations (Beylkin, 1992). In 1982, Jean Morlet (Young, 1995), a French geophysical engineering, first introduced the idea of the wavelet transform as a new mathematical tool for seismic data analysis. The wavelet transform decomposes a signal into a representation that shows signal details and trends as a function of time (Lokenath, 1998). However, the wavelet transform suffers from orientation selectivity in edge data of higher dimensional signals.

In 1999, an anisotropic geometric wavelet transform, named the ridgelet transform, was proposed by Candes and Donohe (1999). To analyze local line or curve singularities, an image or signal is partitioned into subimages, and then to the ridgelet transform is applied to the so obtained sub-images. This block ridgelet based transform, named the curvelet transform, was first proposed in 2000 and underwent several modifications (Candes and Donoho, 2005). The emergence of the curvelet transform has overcome the problem of orientation selectivity encountered in wavelet analysis when used in in feature representation in different areas of signal/image processing, fusion applications like satellite imaging, remote sensing, and multi-focus imaging. The curvelet transform exhibits good reconstruction of the edge data as it incorporates a directional component to the conventional wavelet transform and therefore can be robustly used in analysis of higher dimensional signals (Bhosale, 2014). The curvelet transform thus proved more efficient than all other multiscale transforms including the wavelet transform in signal/image processing applications like signal filtering, enhancement, compression, denoising and watermarking (Kota and Reddy, 2011).

This paper evolves a full-fledged analytical framework for the curvelet transform constructed through wavelet analysis, presenting a computational relationship between curvelets and wavelets. Employing this framework, a new signal denoising model is formulated. Simulations are performed with the radar signal data as a sample multispectral data and, in the end, performance factor analysis is carried out to compare the efficiency of curvelets over wavelets.

\section{PREREQUISITES}

\subsection{Wavelet Transform}

The classical wavelet transform, also known as the continuous wavelet transform (CWT), is a decomposition of a function $f(x)$, with respect to a basic wavelet $(x)$, given by the convolution of a function with a scaled and translated version of $\Psi(x)$ as

$$
\begin{aligned}
& W_{\Psi}(a, b)[f]=|a|^{-\frac{1}{2}} \int f(x) \Psi^{*}\left(\frac{x-b}{a}\right) d x \\
& =\left\langle f, \frac{1}{\sqrt{|a|}} \Psi\left(\frac{x-b}{a}\right)>=\left\langle f, \Psi_{a, b}\right\rangle=<f, U(a, b)>\right. \\
& =W_{\Psi} f(a, b),
\end{aligned}
$$

where $<\ldots, \ldots>$ is the inner product. The range of integral is entire $R^{n}$. The functions $f$ and $\psi$ are square integrable functions and $\psi$ satisfies the admissibility condition

$$
C_{\Psi}=\int \frac{|\widehat{\Psi}(w)|^{2}}{|w|} d w<\infty
$$

Here $c_{\Psi}$ is called the admissibility constant. The superscript * denotes complex conjugation, ' $a$ ' is the scale parameter, $(a>0)$, and ' $b$ ' is the translation parameter. The term $\frac{1}{\sqrt{|a|}}$ is the energy conservation term that keeps energy of the scaled mother wavelet equal to the energy of the original wavelet. The function $f(x)$ can be recovered by the reconstruction formula called the inverse wavelet transform,

$$
f(x)=\frac{1}{C_{\Psi}} \iint W_{\Psi} f(a, b) \frac{1}{\sqrt{|a|}} \Psi\left(\frac{x-b}{a}\right) \frac{d a d b}{a^{2}},
$$

where the admissibility constant $C_{\Psi}>0$. The spectral representation of CWT is obtained by making substitution for $f(x)$ usingthe inverse Fourier transform,

$f(x)=\frac{1}{2 \pi} \int_{-\infty}^{\infty} \exp (i w x) \hat{f}(w) d w$, in $(1)$ as 
$W_{\Psi}[f(x)](a, b)=\frac{1}{2 \pi}|a|^{1 / 2} \int_{-\infty}^{\infty} \hat{f}(w) \exp (i w b) \overline{\widehat{\Psi}(a w)} d w$

In dyadic form, choosing scaling function as power of two, the discrete wavelet, $\psi_{m . n}(t)=2^{-\frac{m}{2}} \psi\left(2^{-m} t-\right.$ $n$ ), is used in multiresolution analysis that constitutes an orthonormal basis for $L^{2}(R)$ (Young, 1995).

\subsection{Curvelet transform}

The curvelet transform is defined as

$C_{j, k, l}(f)=\int_{R^{2}} f(x) \overline{\gamma_{j, k, l}}(x) d x=\int_{R^{2}} \hat{f}(\xi) \overline{\hat{\gamma}_{j, k, l}}(\xi) d \xi$,

where $\hat{f}(\xi)=\frac{1}{2 \pi} \int_{R^{2}} f(x)^{e^{-i<x, \xi>}} d x$,

$\hat{\gamma}_{j, k, l}(\xi)=e^{-i<b_{k}^{j, l}, \xi>} U_{j}\left(R_{\theta_{j, l}}, \xi\right)=e^{-i b_{k}^{j, l} \xi} 2^{-\frac{3}{4} j} W\left(2^{-j} r\right) V \frac{\left(w+\theta_{j, l}\right)}{\theta_{j, l}}(7)$

where $W(r), V(r)$ are window functions satisfying the conditions given below.

$\int_{0}^{\infty} W(a r)^{2} \frac{d a}{a}=1, \forall r>0, \int_{-1}^{1} V(t)^{2} d t=1$

\section{CURVELET TRANSFORM IN TERMS OF WAVELET TRANSFORM}

It is obtained by making substitutions for $\hat{f}(\xi)$ and $\hat{\gamma}_{j, k, l}(\xi)$ as given in (6) and (7) in (5),

$$
C_{j, k, l} f(x)=\frac{1}{2 \pi} \int_{R^{2}} e^{-i x \xi} e^{i b_{k}^{j, l} x} U_{j}\left(R_{\theta_{j, l}}, x\right) f(x) d x
$$

$=\frac{1}{2 \pi} \int_{R^{2}} e^{-i x \xi} e^{i s x} U_{j}\left(R_{\theta_{j, l}}, x\right) f(x) d x$, wheres $=b_{k}^{j, l}$

$=\frac{1}{\sqrt{|a|}} \frac{1}{2 \pi}|a|^{1 / 2} \int_{-\infty}^{\infty} e^{i w x} \hat{\psi}(x) f(x) d x$, where $w=s-\xi$

Using $\hat{\psi}(x)=\exp \left(i \pi x^{\wedge} 2\right)=\exp \left[i \pi\left(\frac{x-b}{a}\right)^{2}\right]$ as the analyzing wavelet,

$$
\begin{aligned}
C_{j, k, l} f(x) & =\frac{1}{\sqrt{|a|}}\left[\frac{1}{2 \pi}|a|^{\frac{1}{2}} \int_{-\infty}^{\infty} e^{i w x} \exp \left[i \pi\left(\frac{x-b}{a}\right)^{2}\right]\right] f(x) d x \\
& =\frac{1}{\sqrt{|a|}}\left[\frac{1}{2 \pi}|a|^{\frac{1}{2}} \int_{-\infty}^{\infty} e^{i\left[w x+\pi\left(\frac{x-b}{a}\right)^{2}\right]}\right] f(x) d x \\
=\frac{1}{\sqrt{|a|}} W_{\Psi}[f(x)](a, b) &
\end{aligned}
$$

Thus, Curvelet Transform $=\frac{1}{\sqrt{|a|}}$ (WaveletTransform $)$, for a particular analyzing wavelet $\psi$ satisfying the admissibility condition (2), where the functions $f$ and $\psi$ are square integrable functions

\section{ANALYTICAL BEHAVIOR OF CURVELET TRANSFORM}

\subsection{The test function space $S$}

An infinitely differentiable complex valued function $\phi$ on $R^{n}$ is said to belong to the test function space $S\left(R^{n}\right)$ if

$\gamma_{v, \beta}(\phi)=\sup _{x \in R^{n}}\left|D^{\beta} \phi(x)\right|<\infty$, for all $\beta \in N_{0}^{n}$.

The dual space of $S$, is $S^{\prime}$, the space of tempered distributions.

\subsection{Generalized Curvelet Transform}

The distributional curvelet transform of $f(x) \in S^{*}\left(R^{n}\right)$ is defined by

$C_{j, k, l}\{f(x)\}=C_{j, k, l}(w, a, b)=\left\langle f(x), K\left(x, w, a_{,}, b\right)\right.$, 
where $K(x, w, a, b)=\frac{1}{\sqrt{|a|}}\left[\frac{1}{2 \pi}|a|^{\frac{1}{2}} \int_{-\infty}^{\infty} e^{i\left[w x+\pi\left(\frac{x-b}{a}\right)^{2}\right]}\right] f(x) d x$, and $K_{\alpha}(x, a,, b) \in S, f \in S^{*}$

\subsection{Analyticity Theorem}

Let $f(x) \in S^{\prime}$ and its curvelet transform $C_{j, k, l}(w, a, b)$ be as defined in (9). Then, $C_{j, k, l}(w, a, b)$ is analytic for some fixed $a, b$ and $w \in \Omega$, where $\Omega_{f}=\left\{w: \sigma_{1}<\operatorname{Re} w<\sigma_{2}\right\}$, and

$D_{w} C_{j, k, l}(w, a, b)=\frac{\partial}{\partial w} C_{j, k, l}(w, a, b)=\left\langle f(x), \frac{\partial}{\partial w} e^{i\left[w x+\pi\left(\frac{x-b}{a}\right)^{2}\right]}\right\rangle$.

For, let $w$ be an arbitrary but fixed point in $\Omega_{\mathrm{f}}$. Choose the real positive numbers $p, q, r$, such that $\sigma_{1}<p<$ $w-r<w+r<q<\sigma_{2}$. Also let $\Delta w$ be a complex increment such that $0<|\Delta w|<r$. Now consider

$$
\begin{aligned}
\frac{C_{j, k, l}(\mathrm{w}+\Delta w, a, b)-C_{j, k, l}(w, a, b)}{\Delta p}-\left\langle f(x), \frac{\partial}{\partial w} e^{i\left[w x+\pi\left(\frac{x-b}{a}\right)^{2}\right]}\right\rangle \\
=\left\langle f(x), \frac{1}{\Delta w}\left[\left(e^{i\left[(w+\Delta w) x+\pi\left(\frac{x-b}{a}\right)^{2}\right]}\right)-\left(e^{i\left[w x+\pi\left(\frac{x-b}{a}\right)^{2}\right]}\right)\right]-\frac{\partial}{\partial w}\left(e^{i\left[w x+\pi\left(\frac{x-b}{a}\right)^{2}\right]}\right)\right\rangle \\
=\left\langle f(x), \psi_{\Delta w}(x)\right\rangle,
\end{aligned}
$$

where

$$
\psi_{\Delta w}(x)=\frac{1}{\Delta w}\left[\left(e^{i\left[(w+\Delta w) x+\pi\left(\frac{x-b}{a}\right)^{2}\right]}\right)-\left(e^{i\left[w x+\pi\left(\frac{x-b}{a}\right)^{2}\right]}\right)\right]-\frac{\partial}{\partial w}\left(e^{i\left[w x+\pi\left(\frac{x-b}{a}\right)^{2}\right]}\right)
$$

Now, to show $\psi_{\Delta w}(x) \in C_{j, k, l}(w, a, b)$, it is enough to show that, as $|\Delta w| \rightarrow 0, \psi_{\Delta w}(x)$ converges in $C_{j, k, l}(w, a, b)$ to zero. Let $c$ denote a circle with centre at $p$ and radius $r_{1}$ where $0<r<r_{1}<\min (w-d)$. Interchanging differentiation on $w$ with differentiation on $x$ and by using the Cauchy integral formula,

$$
\begin{aligned}
&\left(-D_{x}^{m}\right) \psi_{\Delta w}(x)= \frac{1}{\Delta w}\left\{\sum_{k=0}^{m}\left(\begin{array}{l}
m \\
k
\end{array}\right) \sum_{j=0}^{k} C_{k}(i)^{m-j}(2 x-2 b)^{k-2 j} e^{(k-j) p}(w+\Delta w)^{m-k} e^{[(k-j)+i x](w+\Delta w)}\right. \\
&-\sum_{k=0}^{m}\left(\begin{array}{c}
m \\
k
\end{array}\right) \sum_{j=0}^{k} C_{k}(i)^{m-j}(2 x-2 b)^{k-2 j} e^{(k-j) p}(w)^{m-k} e^{[(k-j)+i x] w} \\
&\left.-\frac{\partial}{\partial p}\left[\sum_{k=0}^{m}\left(\begin{array}{c}
m \\
k
\end{array}\right) \sum_{j=0}^{k} C_{k}(i)^{m-j}(2 x-2 b)^{k-2 j} e^{(k-j) p}(w)^{m-k} e^{[(k-j)+i x] w}\right]\right\} \\
& D_{x}^{m} \psi_{\Delta w}(x)=\frac{e^{-s t} \Delta p}{2 \pi i} \int_{c}\left[\frac{p(-z-p) a^{2 z} x^{-z-q-1}-p(z-q) x^{z-q}}{(z-p-\Delta p)(z-p)^{2}}\right] d z
\end{aligned}
$$

Now for all $z \in c, 0<x<\infty, \underset{I}{\sup }\left|D_{x}^{m} \psi_{\Delta w}(x)\right| \leq N$, for some constant $N$, where $N$ is a constant independent of $z$ and $x$.

Moreover, $|z-w-\Delta w|>r_{1}-r>0$ and $|z-w|=r_{1}$

Consequently, $\sup \left|D_{x}^{m} \psi_{\Delta w}(x)\right|=\sup \left|\frac{\Delta w}{2 \pi i} \int_{c}\left[\frac{p(-z-q) a^{2 z} x^{-z-q-1}-p(z-q) x^{z-q}}{(z-w-\Delta w)(z-w)^{2}}\right] d z\right| \leq \frac{|\Delta w| C_{2}}{\left(r_{1}-r\right)}$

Since the right hand side of equation (12) is independent of $x$ and converges to zero as $|\Delta w| \rightarrow 0, \psi_{\Delta w}(x)$ converges toz.

\subsection{Inversion Theorem}

Let $f(x) \in S\left(R^{n}\right), \quad 0<\alpha \leq \frac{\pi}{2} \quad$ and $\quad \operatorname{supp} f \subset S_{d}$, where $\quad S_{d}=\left\{x: x \in R^{n},|x| \leq d, d>0\right\}$ and $\quad$ let $C_{j, k, l}(w, a, b)$ be the distributional Curvelet transform of $f$ as defined by

$\{f(x)\}=C_{j, k, l}(w, a, b)=\langle f(x), K(x, w, a,, b)\rangle$, where

$\mathrm{K}\left(x, w, a_{,}, b\right)=\frac{1}{\sqrt{|a|}}\left[\frac{1}{2 \pi}|a|^{\frac{1}{2}} \int_{-\infty}^{\infty} e^{i\left[w x+\pi\left(\frac{x-b}{a}\right)^{2}\right]}\right] f(x) d x$, 
then for each $\emptyset(x) \in S\left(R^{n}\right)$,

$\lim _{p \rightarrow \infty}\left\langle\frac{1}{2 \pi} \int_{-P}^{P} \widetilde{K}(x, w, a, b) F_{\alpha}(w) d u, \emptyset(x)\right\rangle=\langle f(x), \emptyset(x)\rangle$

where $\widetilde{K}((x, w, a, b))=e^{-i\left[w x+\pi\left(\frac{x-b}{a}\right)^{2}\right]}$.

\section{APPLICATIONS: RADAR/SATELLITE SIGNAL DENOISING}

A signal or an image is usually contaminated by various factors during acquisition or transmission causing noisy effects at the receiving end. These noisy effects decrease the performance of visual and computerized analysis. For both types of radar,continuous wave radar (that continuously transmits a high-frequency signal) and pulse radar (that transmits high power, high-frequency pulses toward the objects and waits for the echo of the transmitted signal before it transmits a new pulse), the quality of such transmitted signals is distorted in a noisy environment. The noise removal or the denoising process is aimed at removing the noise with the help of a matched filter without distorting the quality of processed signal or image. The process, either based on wavelet or curvelet as matched filter, consists of three major steps: decomposition of the transmitted signal, thresholding to demise noisy elements and reconstruction of the processed signal.

The mathematical formulation of the signal $x(t)$ contains two components and is expressed as $x(t)=$ $s_{i}(t)+n_{i}(t)$, where $s_{i}(t)$ represent signal of interest (e.g. object) and $n_{i}(t)$ represents noise factor. The spectrum of the transmitted signal $s_{i}(t)$ is $F_{s_{i}}(\omega)=\int_{-\infty}^{\infty} s_{i}(t) e^{-i \omega t} d t$, the Fourier transform of $s_{i}(t)$ and the noise $n_{i}(t)$ is generally additive white Gaussian noise whose power spectrum is N/2(Saiful et al., 2013).

The decomposition of the signal with matched filter, say, $h(t)$ (in this case wavelet or curvelet based) yields the output, $y(t)=s_{0}(t)+n_{0}(t)$ so as to generate a peak ratio of $s_{0}(t)$ and $n_{0}(t)$ in the sampling values at time $T$. Note that the resulted output, $y(t)$, also contains two components that represent the transmitted signal and the noise respectively, where the component, $s_{0}(t)$ is $s_{o}(t)=\int_{-\infty}^{\infty}\left[H(\omega) F_{S}(\omega)\right] e^{i \omega t} d \omega$ and the noise component, $n_{0}(t)$, is a Gaussian distribution. The matched filter maximizes the peak signal to noise ratio (PSNR), the ratio of the power of $s_{\mathrm{o}}(t)$, and the power of $n_{\mathrm{o}}(t)$ according to the Schwarz inequality.

Decomposition process begins with application of a wavelet filter (with low-pass filter $h$, high-pass filterg, and down sampling by a factor of 2 at each stage of the filter bank), as a result of which, the given signal, $x(t)$, is decomposed into low and high frequency components, termed as approximation coefficients and detail coefficients respectively. The low pass and high filters are given by $h(n)=2^{\frac{-1}{2}}\langle\varphi(t), \varphi(2 t-1)\rangle, g(n)=2^{\frac{-1}{2}}\langle\psi(t), \varphi(2 t-1)\rangle=(-1)^{n} h(1-n)$

As an intermediate step, wavelet coefficients are converted into curvelet coefficient by using (8).

Next, a thresholding technique is applied to remove the noise component from the decomposed signal data that appears in curvelet coefficient form. Thresholding is a simple operation and is performed by selecting the coefficients below a certain threshold and setting them to zero as

$c_{\lambda}=\left\{\begin{array}{c}c_{\lambda},\left|c_{\lambda}\right| \geq t_{\lambda} \\ 0,\left|c_{\lambda}\right|<t_{\lambda}\end{array}\right.$

where $c_{k}$ are the curvelet coefficients, $t_{\lambda}$ is the threshold, $\lambda$ being the index. The threshold value is adjusted using $t_{\lambda}=\sigma \sqrt{2 \log } L, \sigma$ is a noise variance computed using $\sigma=\frac{\left.\operatorname{Median}\left(d_{-}(L-1, k)\right)\right)}{0.6745}$, where $L=N^{2}$, the size of the signal (Yaser and Mahdi, 2011).The thresholding process leads to shrinking the noisy coefficients in the threshold interval $\left[-t_{\lambda}, t_{\lambda}\right]$ and retaining the detail coefficients.

Finally, the signal $(t)$, in turn, is reconstructed as

$x(t)=\frac{1}{\sqrt{|k|}} \sum_{m=1}^{L}\left[\sum_{k=-\infty}^{\infty} D_{m}(k) \psi_{m, k}(t)+\sum_{k=-\infty}^{\infty} A_{l}(k) \varphi_{m, k}(t)\right]$,

where,$\psi_{m, k}(t)$ is the discrete analysing wavelet, $\varphi_{m, k}(t)$ is the discrete scaling, $D_{m}(k)$ is thedetailed signal at scale $2^{m}$, and $A_{l}(k)$ is the approximated signal at scale $2^{l} . D_{m}(k)$ and $A_{l}(k)$ are obtained by applying the scaling and wavelet filters (Mallat, 1999).

To facilitate comparison in terms of efficiency of curvelets over wavelets, performance factor analysis is carried out by using the statistical measures, such as, peak signal to noise ratio (PSNR) and root mean square error (RMSE), computed as 
$P S N R=10 \log _{10}\left(\frac{\sum_{i=1}^{N} x^{2}(i)}{\sum_{i=1}^{N}[x(i)-\hat{x}(i)]^{2}}\right), R M S E=\frac{1}{N} \sqrt{\sum_{i=1}^{N}[x(i)-\hat{x}(i)]^{2}}(16)$

where $x(i)$ is the original source signal, $\hat{x}(i)$ is the separated signal, $i$ is the sample index and $N$ is the number of samples of the signal.A better performance of the matched filter is shown by a higher the value of PSNR with minimum value of RMSE.To demonstrate the performance of the proposed model, multispectral image sample data is considered.

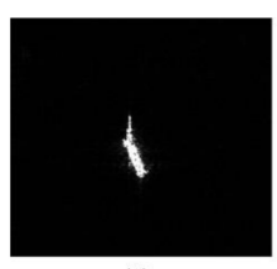

(a)

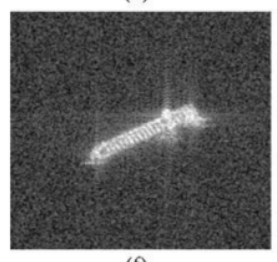

(f)

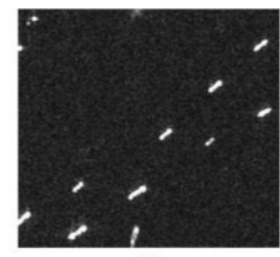

(b)

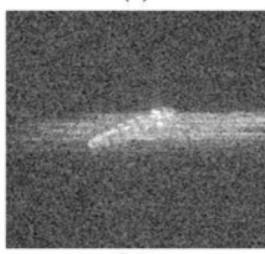

(g)

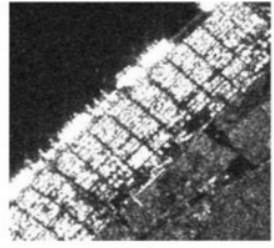

(c)

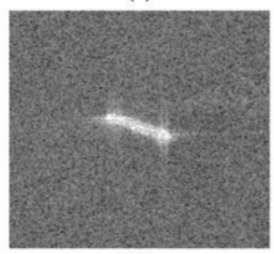

(h)

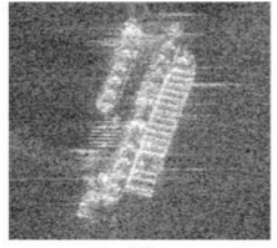

(d)

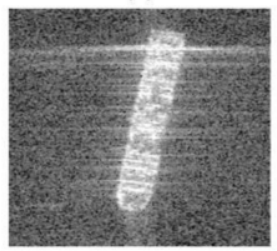

(i)

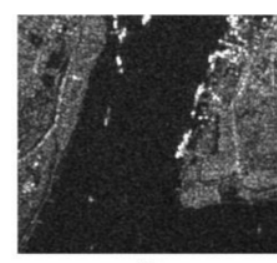

(e)

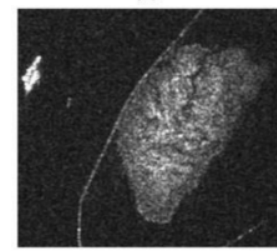

Figure 1. Some examples of ship chips. $(\mathbf{a}, \mathbf{h})$ are the cropped ship chips from the Gaofen-3 images with FS1 imaging mode. (b,c,e,j) are the cropped ship chips from the Sentinel-1 images with Interferometric Wide (IW) imaging mode. (d,f,g,i) are the cropped ship chips from the Gaofen-3 images with Ultrafine Strip (UFS) imaging mode (Wang, et al., 2019)

In a multispectral dataset, the band information is reported as the centre wavelength value that represents the centre point value of the wavelengths. Simulations are performed on noisy mixed sample signal data on Matlab® R 7.9 on a core $i 72.2 \mathrm{GHz}$ PC using the USFFT software package.

The results (PSNR in $\mathrm{dB}$ ) along with RMSE are presented below:

Table 1. PSNR in $\mathrm{dB}$

\begin{tabular}{|l|l|l|l|l|}
\hline Image & Signal data & $\begin{array}{l}\text { Wavelet } \\
\text { thresholding }\end{array}$ & Curveletthresholding & RMSE \\
\hline Sample I & $\begin{array}{l}20.01(\mathrm{~m}=0, \\
\left.\sigma^{2}=0.01\right)\end{array}$ & 24.89 & 26.15 & 0.1214 \\
\hline Sample II & $\begin{array}{l}21.09 \quad(\mathrm{~m}=0, \\
\left.\sigma^{2}=0.01\right)\end{array}$ & 26.89 & 27.14 & 0.1234 \\
\hline Sample III & $\begin{array}{l}19.77(\mathrm{~m}=0, \\
\left.\sigma^{2}=0.01\right)\end{array}$ & 27.70 & 29.15 & 0.0764 \\
\hline
\end{tabular}

\section{DISCUSSION AND CONCLUSION}

In this work, the curvelet transform has been presented as an extension of the wavelet transform by exploiting the relationship between them, and a full-fledged analytical framework has been established in new settings. In order to avoid computational complexity, instead of computing curvelet coefficients, the wavelet coefficients are computed first and then curvelet coefficients are obtained using the relationship between them. Thus, the curvelet expressed in terms of the wavelet has been employed more conveniently in the proposed signal denoising model without loss of signal edge information. As an illustration, the performance factor analysis is conducted with the sample data.

The results show that besides gaining on the computational complexity, the proposed curvelet based model has better performance in terms of increased PSNR with minimized RMSE than the one based on wavelets. The proposed model can be applied to both pulse signal as well as the image, since the image can be represented as two-dimensional signal. 


\section{REFERENCES}

Bhosale, B (2014), Wavelet Analysis of randomized Solitary wave solutions, Int. J. Math Analysis andApplications, 1(1), 20-26.

Bhosale, B (2014), Curvelet Based Multiresolution Analysis of Graph Neural Networks, Int. J Applied Physics and Mathematics, 14 (5), 313-323.

Bhosale, B., and A. Biswas (2013), Multi-resolution analysis of wavelet like soliton solution of $\mathrm{KdV}$ equation, International Journal of Applied Physics and Mathematics, 3(4), 270-274.

Beylkin, G (1992), On the representation of operators in bases of compactly supported wavelets, SIAM J.Numerical Analysis, 6, 1716-1740.

Candes, E., and D. Donoho (1999), Ridgelets: A key to higher dimensional intermittency, Philos Trans R. Soc.London A, Math, Phys, Eng. Sci, 357(1760), 2495-2509.

Candes, E., and D. Donoho (2005), Continuous curvelet transform:Resolution of the wavefront set,Applied and Computational Analysis, 19 (2), 162-197.

Kota, N., and G. Reddy (2011), Fusion Based Gaussian noise removal in the images using Curvelets and Wavelets with Gaussian filter, Int J of Image Processing, 5(4), 230-238.

Lokenath, D (1998), Wavelet transform and their applications, PINSA-A,64 (A)(6), 685-713.

Mallat, S. G. (1999), A wavelet tour of signal processing, Academic Pr.

SaifulI MD, Hyungseob H, Myug L., and J. Gook (2013), Small target detection and noise reduction inmarine radar system, IERI Procedia, 51, 168-173.

Wang, Y., Wang, C., Zhang, H., Dong, Y and S. Wei (2019), A SAR Dataset of Ship Detection for Deep Learning under Complex Backgrounds, Remote Sens,11(7), 765;https://doi.org/10.3390/rs11070765

Yaser, N., and J. Mahdi (2011), A Novel CurveletThresholding Function for Additive Gaussian Noise Removal, International Journal of Computer Theory and Engineering, 3(4), 169-178.

Young, Randy K (1995), Wavelet theory and its applications, Kluwer Academic Publishers. 